\title{
ORIGINAL
}

\section{DISTRIBUCIÓN GEOGRÁFICA DE LOS MÉDICOS EN ESPAÑA Y SU EVOLUCIÓN TEMPORAL DURANTE EL PERÍODO 1998-2007}

\author{
Miguel Ángel García-Pérez (1,2), Carlos Amaya (1), Mª Rosario López-Giménez (2) y Ángel Otero (2) \\ (1) Fundación CESM. Madrid. \\ (2) Departamento de Medicina Preventiva y Salud Pública. Facultad de Medicina, Universidad Autónoma. Madrid.
}

\section{RESUMEN}

Fundamento: La distribución geográfica de los profesionales sanitarios condiciona la accesibilidad de la población a la asistencia sanitaria. El presente estudio pretende analizar la distribución provincial de los médicos en España, su evolución temporal y los factores relacionados con la misma.

Métodos: Estudio observacional, transversal, ecológico, descriptivo y analítico, a partir de fuentes oficiales de datos (Instituto Nacional de Estadística y Ministerio de Sanidad y Consumo). Se obtuvieron, a nivel provincial, variables relativas a la distribución de médicos, condiciones socioeconómicas, estructura sanitaria y capacidad de formación de médicos. Se construyeron distintos estadísticos de variabilidad para la distribución de médicos, y se analizaron las correlaciones bivariadas y multivariadas entre la ratio provincial de médicos y el resto de variables.

Resultados: La razón entre los valores extremos de la variable ratio provincial de médicos en 2007 es de 2,109 $(2,158$ en 1998), el coeficiente de variación de 0,19 ( 0,185 en 1998) y el índice de Gini de 0,103 (0,097 en 1998). La distribución de médicos y la formación especializada provincial están fuertemente relacionadas, tanto en el análisis bivariado $(r=0,87)$, como en el multivariado, en el que las variables socioeconómicas y de estructura sanitaria y la formación especializada explican un $83,3 \%$ de la varianza en la ratio provincial de médicos.

Conclusiones: La variabilidad en la distribución geográfica de médicos en España se ha mantenido prácticamente estable en la última década, siendo similar a la existente en los países de nuestro entorno. La capacidad formativa de cada provincia parece jugar un papel fundamental en la distribución geográfica de los médicos.

Palabras clave: Planificación sanitaria. Recursos humanos en salud. Distribución de médicos. Factores socioeconómicos. Educación médica.

Correspondencia:

Miguel Ángel García Pérez.

Fundación CESM.

C/ Veneras 9, 4. 28013 Madrid.

Correo electrónico: mangel@cesm.org

\section{ABSTRACT}

\section{Physicians' Geographical Distribution in Spain and its Evolution During the Period 1998-2007}

Background: Population accessibility to health care depends on the geographical distribution of health care professionals. The aim of this study is to analyse the provincial distribution of Spanish physicians, its recent evolution and the factors related with it.

Methods: Observational, cross-sectional, ecological, descriptive and analytical study on data from public oficial data sources (Statistics National Institute, Health and Consume Ministry). We obtained, on a provincial basis, variables related with physicians' distribution, socioeconomic conditions, health care resources and medical training capacity. We built some variability indicators for physicians' distribution, and we analysed the bivariated and multivariated correlations between provincial physicians' rate and the other variables.

Results: The ratio between the upper and lower values of the population based physicians' provincial ratio is 2,109 $(2,158$ in 1998), the variation coefficient is $0,19(0,185$ in 1998), and the Gini index is 0,103 (0,097 in 1998). There is a strong correlation between physicians' distribution and the specialist training capacity of each province in bivariated $(\mathrm{r}=0,87)$ and multivariated analysis (where socioeconomic and health care structure indicators and postgraduate training capacity explain $83,3 \%$ of physicians' distribution variance).

Conclusions: The variability of physicians' distribution in Spain has been almost steady in the last decade, and its level is similar to that in other European countries. The provincial training capacity seems to play a fundamental role in physicians' distribution.

Key words: Health planning. Health manpower. Socioeconomic factors. Education, medical. 


\section{INTRODUCCIÓN}

La asistencia sanitaria se basa en la prestación de servicios personales, por lo que el número y la distribución geográfica de los profesionales que la prestan, así como el reparto de competencias y responsabilidades entre ellos, son factores determinantes en la accesibilidad de la población a los servicios sanitarios $^{1-4}$.

En este sentido, el panorama en España está sufriendo un giro inesperado, al haberse pasado de una situación con un número excesivo de médicos ${ }^{5-8}$ a otra en la que existen dificultades crecientes para encontrar profesionales de algunas especialidades y en diferentes áreas geográficas de nuestro país ${ }^{9,10}$. Sin embargo, no hay acuerdo en cuanto a etiquetar la situación como deficitaria, y en diversos medios se pone el acento en la mala distribución de los médicos ${ }^{11}$, que se podría definir como la falta de adecuación entre la distribución geográfica y por especialidades de los profesionales y la distribución geográfica de la población destinataria de sus servicios y sus necesidades de atención.

Más allá de la constatación meramente cuantitativa de una desigual distribución de los profesionales en relación a la población ${ }^{5,10-12}$, se han realizado algunos estudios, restringidos al ámbito provincial o de alguna Comunidad Autónoma ${ }^{13,14}$. Tan sólo conocemos uno, realizado en el año 1979, que haya afrontado específicamente el análisis de los factores relacionados con dicha distribución a nivel nacional, tomando como unidad geográfica de análisis la provincia; en él se concluía que factores como la capacidad formativa de profesionales, la situación económica o algunos factores sociodemográficos influían en la distribución provincial de los facultativos, y que lo hacían de forma diferenciada en las mitades norte y sur de la península ${ }^{15}$. Dado el tiempo transcurrido, se hace necesario actualizar el análisis de este fenómeno.
El presente trabajo analiza la distribución actual de los médicos en nuestro país, su evolución en los últimos años, y los factores relacionados con la misma, bajo la hipótesis inicial de que las características socioeconómicas de cada provincia, sus recursos sanitarios o su capacidad de formación de médicos contribuyen a explicar la distribución geográfica de los profesionales.

\section{SUJETOS Y MÉTODOS}

Diseño del estudio. Se trata de un estudio observacional, transversal, ecológico, descriptivo y analítico, de la distribución actual de los médicos en España y los factores que se asocian con ella, que se complementa con un análisis de la evolución de dicha distribución en los últimos diez años. Tanto la recogida de datos como su análisis se realizaron durante el primer semestre del año 2008.

Unidad de análisis. Para el análisis se eligió como unidad geográfica la provincia, debido a que es el nivel geográfico de menor extensión sobre el que existe información de la mayoría de indicadores necesarios para el estudio. Se trata, además, de ámbitos geográficos de extensión más homogénea que las Comunidades Autónomas (CCAA), y centrados en torno a uno (o dos) centros de atracción, lo que también ofrece una mayor homogeneidad estructural que las CCAA.

Variable principal. La distribución geográfica de los médicos se determinó mediante la ratio provincial de médicos por cien mil habitantes, obtenida a partir de los datos del Instituto Nacional de Estadística $^{16}$, que recoge anualmente los datos de los colegios provinciales, con fecha de referencia a 31 de diciembre de cada año, y los datos oficiales de población a 1 de enero. Dicha ratio se calculó, por tanto, con los datos de total de cole- 
giados a 31 de diciembre de 2006 (último dato existente en el momento de realizar el estudio) y de población a 1 de enero de 2007.

Variables explicativas. Se incluyeron en el estudio variables relacionadas con las condiciones socioeconómicas de cada provincia, su estructura sanitaria y su capacidad docente (entendida como su capacidad para la formación de médicos).

Condiciones socioeconómicas. Para conocer las condiciones socioeconómicas se escogieron indicadores habitualmente utilizados por el Instituto Nacional de Estadística en su página web ${ }^{16}$, con la única limitación de que se ofrecieran a nivel provincial. En este sentido, se obtuvieron los datos de PIB y Renta Disponible Bruta per cápita para el año 2005, y de tasas de paro y de estudios superiores para el 2006; en el caso de las tasas de paro, los datos correspondientes a Ceuta y Melilla se presentaban agrupados, por lo que no se pudieron independizar sus valores en cada caso. En relación a la oferta general de servicios estaban disponibles la tasa de unidades docentes de la educación no universitaria y de salas de cine. Como indicadores demográficos, además de la población oficial se obtuvieron también la distribución por grandes grupos de edad (0-14, 15-64 y mayores de 65 años) y la densidad de población, y se construyeron indicadores de distribución de la población provincial según el tamaño de los municipios (porcentaje de población de la provincia que reside en núcleos menores de 2.000 y de 10.000 habitantes y en mayores de 100.000 y de 500.000 habitantes, así como un indicador de distribución de población en municipios urbanos de entre 10.000 y 500.000 habitantes, que excluye tanto el ámbito rural y semi-rural como las grandes urbes). Se decidió, asimismo, añadir una variable que reflejara el incremento de población en los últimos diez años, como porcentaje de la población existente al comienzo de ese intervalo (1997), para valorar la posible influencia de la dinámica poblacional en la variable principal (ratio provincial de médicos).

Estructura sanitaria. La estructura sanitaria de cada provincia se valoró mediante el número de camas por cien mil habitantes, a partir de la Estadística de recursos hospitalarios del Ministerio de Sanidad y Consu$\mathrm{mo}^{17}$.

Capacidad docente. La capacidad docente se calculó mediante tres ratios poblacionales (con la base poblacional correspondiente al año 2007), obtenidas a partir de las siguientes cifras:

- Número total de médicos licenciados en cada provincia, a partir de los datos del Ministerio de Educación (años 1950 - 1998) ${ }^{18}$ y del Consejo de Coordinación Universitaria (años 2004 y 2005) ${ }^{19}$, asignando a los años intermedios la media entre los valores correspondientes a 1998 y 2004.

- Número total de especialistas formados en la correspondiente provincia mediante el sistema MIR, desde su inicio, a partir de las bases de datos utilizadas para el estudio Demografía médica en España. Mirando al futuro ${ }^{20}$;

- Número de plazas MIR ofertadas en la convocatoria 2004. Esta variable se utiliza para valorar la capacidad formativa puntual de cada provincia y la influencia que pudieran tener los médicos en formación sobre la cantidad total de médicos en la provincia.

Cuando fue necesario obtener un indicador de base poblacional, se puso en relación con la cifra oficial de población a 1 de enero del año siguiente, dando los resultados en unidades por cien mil habitantes. 
Otras variables. Se incluyeron las variables de longitud y latitud de la capital de provincia para poder analizar la posible persistencia de varianza residual relacionada con las mismas y no explicable por otras variables del modelo, como ya ocurrió en un estudio previo ${ }^{15}$. Asimismo, y para valorar la posible influencia de la existencia o no de colegiación obligatoria sobre la variable principal, se incorporó una variable cualitativa que recogiera esta circunstancia; como se sabe; la colegiación no es obligatoria para los médicos empleados exclusivamente en la sanidad pública en las provincias de las CCAA de Andalucía, Extremadura, Canarias y Cantabria.

Análisis estadístico. Determinada la distribución normal de la variable ratio provincial de médicos, se analizó la distribución geográfica de los médicos mediante su media y medidas de dispersión y estadísticos de variabilidad, tales como la desviación estándar de las ratios provinciales, el coeficiente de variación (razón entre la desviación estándar y la media de cada año), la razón entre ratios extremas, la razón de deciles y el índice de Gini (según la fórmula de Brown ${ }^{21}$ ), para el período 1997-2006. También se representaron, de forma gráfica, los cuartiles de distribución de la variable en un mapa provincial de España. Para facilitar la comparación internacional, se calcularon la razón entre ratios extremas y el índice de Gini en el año 2006 a nivel de CCAA

Se procedió al análisis descriptivo de cada una de las variables explicativas, determinando la posible normalidad de su distribución mediante la prueba de Kolmogoroff-Smirnov (con un nivel de significación de $\mathrm{p}<0,05$ ). Se analizaron las diferentes correlaciones bivariadas mediante los coeficientes $\mathrm{r}$ de Pearson (para variables gaussianas) y $\rho$ de Spearman (cuando no se podía asumir la distribución normal). Se analizó asimismo la distribución de la variable colegiación obligatoria, y la posible relación de ésta con la variable principal mediante la prueba t de Student para la comparación de medias en muestras pequeñas.

Para identificar las posibles variables predictoras de la distribución provincial de médicos se realizó un análisis de regresión lineal. Se introdujeron todas las variables con distribución normal, y se eliminaron aquéllas que no tuvieran una aportación significativa en el modelo (procedimiento "hacia atrás" en SPSS); seguidamente, se analizó la aportación de cada una de las variables o grupo de variables que lo constituyen mediante el valor de sus coeficientes parciales de regresión estandarizados, su influencia bruta en el valor de la variable de estudio, y la magnitud y significatividad del cambio en $\mathrm{R}^{2}$ al eliminar cada variable del modelo o sustituirla por otras de su misma categoría.

Finalmente se complementó el análisis de regresión mediante el procedimiento de modelos lineales generalizados para considerar el efecto de las variables de distribución no gaussiana, valorando la significación estadística de su adición a los modelos conseguidos en la regresión lineal mediante el análisis de significatividad de la razón de verosimilitud (menos dos veces la diferencia entre los logaritmos de verosimilitud del modelo inicial y del modelo con nuevas variables) por medio de la prueba de chi cuadrado.

La tabulación y análisis de los datos se realizó mediante el programa estadístico SPSS 15.0, y las imágenes mediante el programa GIMP.

\section{RESULTADOS}

La ratio provincial de médicos por cien mil habitantes en el año 2007 (tabla 1) oscila entre los 631,6 de Salamanca y los 
Tabla 1

Ratio provincial de médicos por cien mil habitantes (1 de enero de 2007)

\begin{tabular}{|l|l|l|l|}
\hline \multicolumn{1}{|c|}{ Provincia } & Ratio & Provincia & Ratio \\
\hline Álava & 480,6 & Lugo & 377,6 \\
\hline Albacete & 379,7 & Madrid & 546,7 \\
\hline Alicante & 331,9 & Málaga & 402,8 \\
\hline Almería & 305,6 & Murcia & 369,5 \\
\hline Ávila & 423,4 & Navarra & 555,7 \\
\hline Badajoz & 416,7 & Ourense & 402,2 \\
\hline Baleares & 421,4 & Asturias & 521,6 \\
\hline Barcelona & 507,2 & Palencia & 475,0 \\
\hline Burgos & 451,1 & Palmas (Las) & 383,1 \\
\hline Cáceres & 412,4 & Pontevedra & 391,8 \\
\hline Cádiz & 360,0 & Salamanca & 631,6 \\
\hline Castellón & 347,3 & Tenerife & 393,8 \\
\hline Ciudad Real & 371,7 & Cantabria & 517,4 \\
\hline Córdoba & 401,9 & Segovia & 465,1 \\
\hline Coruña (A) & 450,8 & Sevilla & 433,1 \\
\hline Cuenca & 317,4 & Soria & 565,2 \\
\hline Girona & 321,3 & Tarragona & 346,7 \\
\hline Granada & 468,5 & Teruel & 467,2 \\
\hline Guadalajara & 390,5 & Toledo & 326,0 \\
\hline Guipúzcoa & 475,6 & Valencia & 480,6 \\
\hline Huelva & 346,8 & Valladolid & 552,7 \\
\hline Huesca & 433,4 & Vizcaya & 520,9 \\
\hline Jaén & 299,5 & Zamora & 440,6 \\
\hline León & 429,6 & Zaragoza & 624,7 \\
\hline Lleida & 367,9 & Ceuta & 347,2 \\
\hline Rioja (La) & Melilla & 313,9 \\
\hline
\end{tabular}

299,5 de Jaén, con un neto gradiente norte-sur, claramente visible en la figura 1. La razón entre ambos valores extremos es de 2,109, habiéndose reducido ligeramente desde 1998, en que su valor era 2,158; sin embargo, la razón entre los deciles superior e inferior, el coeficiente de variación y el índice de Gini han aumentado ligeramente, aunque no de forma continua (tabla 2).

La descripción de las variables estudiadas se muestra en la tabla 3, en la que se aprecia que las variables ratio de licenciados, densidad de población y tasa de población en municipios mayores de 100.000 y 500.000 habitantes no siguen una distribución normal.

La diferencia de medias de la variable principal entre las provincias con y sin colegiación obligatoria (437,2 frente a 395,5 respectivamente) no alcanza significatividad estadística $(\mathrm{p}=0,10)$ mediante la prueba $\mathrm{t}$ de Student.

El análisis bivariado de correlaciones (tabla 4) muestra que la distribución provincial de médicos se correlaciona fuerte-

Figura 1

Cuartiles de distribución de la ratio provincial de médicos en 2007, y valoración de su evolución en los últimos diez años

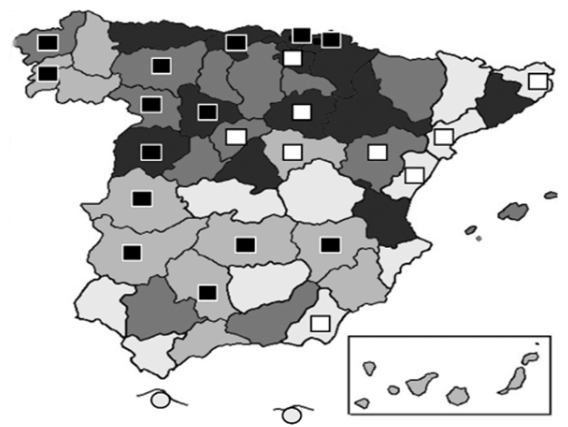

Médicos por cien mil habitantes (color de fondo)

$$
\begin{gathered}
<368 \quad 368-420 \quad 420-475 \square>475 \\
\text { Incremento sobre } 1998 \text { (recuadro) } \\
\square \text { Negativo } \square \text { Positivo }>10 \%
\end{gathered}
$$


Tabla 2

Evolución de la distribución provincial de médicos y su variabilidad en la en los últimos diez años en España

\begin{tabular}{|l|c|c|c|c|c|c|c|c|c|c|c|}
\hline & \multicolumn{9}{|c|}{ Años } \\
\cline { 2 - 12 } & $\mathbf{1 9 9 8}$ & $\mathbf{1 9 9 9}$ & $\mathbf{2 0 0 0}$ & $\mathbf{2 0 0 1}$ & $\mathbf{2 0 0 2}$ & $\mathbf{2 0 0 3}$ & $\mathbf{2 0 0 4}$ & $\mathbf{2 0 0 5}$ & $\mathbf{2 0 0 6}$ & $\mathbf{2 0 0 7}$ \\
\hline Ratio nacional & 422,1 & 426,6 & 431,9 & 435,4 & 431,2 & 435,2 & 441,4 & 441,3 & 445,4 & 449,3 \\
\hline Ratios provinciales & \multicolumn{7}{|c|}{} \\
\hline Media & 402,7 & 407,4 & 411,3 & 415,8 & 412,7 & 417,7 & 422,2 & 421,5 & 423,9 & 426,8 \\
\hline Valor mínimo & 279,8 & 281,1 & 292,7 & 292,7 & 285,2 & 287,7 & 304,7 & 299,6 & 298,6 & 299,5 \\
\hline Valor máximo & 603,8 & 601,6 & 596,2 & 594,7 & 588,7 & 614,7 & 615,0 & 614,2 & 629,8 & 631,6 \\
\hline Desv. típica & 74,3 & 74,6 & 76,1 & 77,1 & 77,9 & 80,2 & 78,6 & 78,3 & 80,5 & 81,0 \\
\hline Coef. variación & 0,185 & 0,183 & 0,185 & 0,185 & 0,189 & 0,192 & 0,186 & 0,186 & 0,190 & 0,190 \\
\hline Razón valores extremos & 2,158 & 2,141 & 2,037 & 2,032 & 2,064 & 2,136 & 2,019 & 2,050 & 2,109 & 2,109 \\
\hline Razón deciles & 1,504 & 1,529 & 1,554 & 1,567 & 1,601 & 1,606 & 1,630 & 1,642 & 1,656 & 1,666 \\
\hline Índice de Gini & 0,097 & 0,100 & 0,098 & 0,098 & 0,095 & 0,096 & 0,097 & 0,099 & 0,102 & 0,103 \\
\hline Ratios por CCAA* & & & & & & & & \\
\hline Razón valores extremos & & & & & & & & & & 1,734 \\
\hline Índice de Gini & & & & & & & & & & 0,079 \\
\hline
\end{tabular}

* Calculadas exclusivamente a efectos de comparación internacional.

mente con la ratio provincial de especialistas formados mediante el sistema de residencia $(r=0,82)$ y la tasa de estudios superiores $(\mathrm{r}=0,62)$. En cuanto al resto de variables, y aparte de algunas correlaciones lógicas de los indicadores socioeconómicos entre sí no mostradas en la tabla, llama la atención la correlación entre la dotación escolar y la distribución por edad de la población, siendo inversa para la población menor de 15 y menor de 65 años, y directa para la población de mayor edad; además, exista una correlación negativa entre dicha dotación escolar y el incremento de población, lo que puede hacer pensar en un posible efecto inercial en la dotación de servicios sobre el que volveremos más adelante.

El análisis multivariante muestra que un modelo con sólo cuatro variables, de las tres categorías analizadas (ratio de especialistas formados, ratio de camas, incremento de población y tasa de paro), explica el 83,3\% de la varianza de la distribución provincial de médicos (tabla 5). La adición de la variable longitud geográ- fica parece incrementar ligeramente el poder predictivo $\left(\mathrm{R}^{2}=0,837\right)$, pero el incremento no es estadísticamente significativo $(\mathrm{p}<0,14)$, por lo que no se ha incluido en el modelo.

La mayor reducción en el porcentaje de varianza predicho $(-45,2 \%)$ se produce cuando se retira la variable de especialistas formados; ella sola explicaría el 64,1\% de la varianza de la distribución de médicos. La retirada aislada de la variable tasa de paro no afecta de forma considerable al modelo, mientras que su intercambio con la tasa de estudios superiores produce un modelo más inestable, y con el indicador de renta disponible bruta per cápita produce un modelo similar al original (predice un $83 \%$ de la varianza de la distribución de médicos) (resultados no mostrados en la tabla). Tasa de paro, tasa de estudios superiores y renta disponible bruta per capita parecen estar aportando una información similar.

Un modelo alternativo, que pretendiera explicar la variabilidad de la distribución 
Tabla 3

Análisis descriptivo de las diferentes variables incluidas en el estudio

\begin{tabular}{|c|c|c|c|c|c|c|}
\hline Variables para las que se asume distribución normal & $\mathbf{N}$ & $\begin{array}{c}\text { Kolmogoroff- } \\
\text { Smirnov, p= }\end{array}$ & Mínimo & Máximo & Media & Desv. típ. \\
\hline Ratio de médicos & 52 & 0,87 & 299,51 & 631,61 & 426,79 & 80,97 \\
\hline PIB per capita & 52 & 0,40 & 13.804 & 29.196 & $19.666,15$ & $3.852,61$ \\
\hline Renta disponible bruta per capita & 52 & 0,32 & $9.950,20$ & $17.232,99$ & $12.681,34$ & $2.015,30$ \\
\hline Tasa de paro & 50 & 0,33 & 4,66 & 17,65 & 9,30 & 3,23 \\
\hline Tasa de estudios superiores & 52 & 0,34 & 9,83 & 33,58 & 19,83 & 5,00 \\
\hline Ratio de unidades escolares & 52 & 0,30 & 3,91 & 6,77 & 5,14 & 0,74 \\
\hline Ratio de salas de cine & 52 & 0,23 & 3,46 & 19,84 & 10,05 & 3,52 \\
\hline Tasa de población en municipios $<2.000$ hab. & 52 & 0,10 & 0,00 & 0,51 & 0,14 & 0,13 \\
\hline Tasa de población en municipios $<10.000$ hab. & 52 & 0,73 & 0,00 & 0,682 & 0,33 & 0,19 \\
\hline Tasa de población en municipios entre 10.000 y 500.000 hab. & 52 & 0,85 & 0,07 & 1,00 & 0,62 & 0,20 \\
\hline Tasa población $<15$ años & 52 & 0,86 & 0,09 & 0,22 & 0,14 & 0,03 \\
\hline Tasa población entre 15 y 64 años & 52 & 0,47 & 0,62 & 0,73 & 0,68 & 0,03 \\
\hline Tasa población > 64 años & 52 & 0,41 & 0,11 & 0,28 & 0,18 & 0,04 \\
\hline Ratio de camas & 52 & 0,53 & 215,57 & 516,50 & 347,22 & 74,45 \\
\hline Ratio de especialistas formados & 52 & 0,11 & 0,00 & 440,62 & 162,00 & 105,91 \\
\hline Ratio de plazas MIR & 52 & 0,60 & 0,00 & 22,15 & 11,03 & 4,53 \\
\hline Incremento población 1997-2007 (\%) & 52 & 0,10 & $-4,21$ & 42,59 & 11,95 & 12,26 \\
\hline Longitud geográfica & 52 & 0,28 & 28,15 & 43,45 & 39,91 & 3,23 \\
\hline Latitud geográfica & 52 & 0,65 & $-16,25$ & 2,82 & $-3,96$ & 3,55 \\
\hline Variables no gaussianas & $\mathbf{N}$ & $\begin{array}{c}\text { Kolmogoroff- } \\
\text { Smirnov, } \mathbf{p}<\end{array}$ & Mínimo & Máximo & Mediana & $\begin{array}{l}\text { Rango inter- } \\
\text { culartilico }\end{array}$ \\
\hline Densidad de población & 52 & 0,01 & 9,08 & $5.341,54$ & 62,78 & $26,27-162,36$ \\
\hline Tasa de población en municipios $>100.000 \mathrm{~h}$. & 52 & 0,05 & 0,00 & 0,75 & 0,30 & $0-0,41$ \\
\hline Tasa de población en municipios $>500.000$ h. & 52 & 0,01 & 0,00 & 0,70 & 0 & $0-0$ \\
\hline Ratio de licenciados formados & 52 & 0,01 & 0,00 & $3.678,35$ & 0 & $0-466,64$ \\
\hline
\end{tabular}

Ratios por cien mil habitantes, excepto la ratio de unidades escolares, expresada en unidades por cien habitantes menores de 15 años. Tasas de población expresadas en tanto por uno, en relación al total de población de la provincia. Tasas de paro y estudios superiores expresadas en porcentaje de la población activa y de la población mayor de 16 años de cada provincia, respectivamente.

provincial de médicos excluyendo la capacidad de formación de especialistas de la provincia, aún alcanzaría a explicar el $61,6 \%$ de la varianza, incluyendo la tasa de estudios superiores, el indicador de distribución provincial de la población y el porcentaje de incremento poblacional de los últimos diez años (tabla 6). La sustitución de la tasa de estudios superiores por la tasa de paro ocasiona una importante reducción del poder predictivo; la adición de esta última, o de las variables PIB $\mathrm{y}$ renta disponible bruta, introduce una gran inestabilidad en el modelo, sin mejorar su poder predictivo (datos no mostrados en la tabla).

La introducción de las variables no gaussianas mediante el procedimiento de modelos lineales generalizados no generó una mejora significativa en el modelo inicial de cuatro variables; sin embargo, la adición de la variable de licenciados formados sí mejoró significativamente la capacidad predictiva del modelo alternativo (razón de verosimilitud 20,26; 
Tabla 4

Tabla de correlaciones bivariadas de las distintas variables estudiadas con la ratio de médicos y con la tasa de unidades escolares en cada provincia

\begin{tabular}{|c|c|c|c|}
\hline & & Ratio médicos & Tasa de unidades escolares \\
\hline \multirow{19}{*}{ 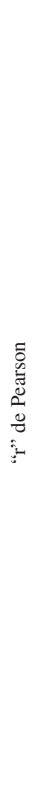 } & Ratio de médicos & 1 & $0,42 *$ \\
\hline & Ratio de especialistas formados & $0,82 *$ & \\
\hline & Ratio de plazas MIR & $0,43 *$ & \\
\hline & Ratio de camas & $0,45^{*}$ & 0,33 \\
\hline & PIB per capita & $0,37 *$ & \\
\hline & Renta per capita & $0,48 *$ & 0,28 \\
\hline & Tasa de paro & $-0,33$ & $-0,32$ \\
\hline & Tasa de estudios superiores & $0,62 *$ & \\
\hline & Tasa unidades escolares & $0,42 *$ & 1 \\
\hline & Ratio de salas cine $\$$ & & \\
\hline & Tasa población $<15$ años & $-0,48 *$ & $-0,82 *$ \\
\hline & Tasa población entre 15 y 64 años & & $-0,65^{*}$ \\
\hline & Tasa población $>64$ años & 0,31 & $0,86^{*}$ \\
\hline & Tasa población en municipios $<2.000$ hab. & & $0,70 *$ \\
\hline & Íd. municipios $<10.000$ & & $0,67 *$ \\
\hline & Íd. municipios entre 10.000 y 500.000 hab. & $-0,32$ & $-0,45^{*}$ \\
\hline & Incremento de población 1997-2007 (\%) & $-0,38 *$ & $-0,68^{*}$ \\
\hline & Longitud geográfica & $0,42 *$ & 0,57 \\
\hline & Latitud geográfica & & \\
\hline \multirow{4}{*}{ 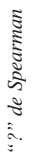 } & Ratio de licenciados formados & $0,47 *$ & \\
\hline & Densidad de población & & $-0,59 *$ \\
\hline & Tasa población en municipios $>100.000$ & $0,37 *$ & $-0,32 *$ \\
\hline & Íd. municipios $>500.000$ & $0,32 *$ & $-0,31$ \\
\hline
\end{tabular}

Sólo se muestran valores con $\mathrm{p}<0,05$. $* \mathrm{p}<0,01$.

Tabla 5

Modelo de regresión lineal múltiple para explicar la ratio provincial de médicos, y análisis de la influencia en el modelo de las distintas variables incluidas en el mismo

\begin{tabular}{|c|c|c|c|c|c|}
\hline \multirow[b]{2}{*}{ Variables } & \multirow{2}{*}{$\begin{array}{c}\text { Modelo } \\
\text { Coeficientes }\end{array}$} & \multicolumn{4}{|c|}{ Valoración de la influencia de cada variable } \\
\hline & & $\begin{array}{c}\text { B } \\
\text { (coeficiente } \\
\text { estandariz) }\end{array}$ & $\begin{array}{c}\text { Aportación media } \\
\text { al valor de la variable de } \\
\text { estudio }\end{array}$ & $\begin{array}{l}\mathbf{R}^{2} \text { del modelo } \\
\text { univariante }\end{array}$ & $\begin{array}{c}\text { Cambio en } \mathbf{R}^{2} \\
\text { al retirar la variable }\end{array}$ \\
\hline Constante & $339,60 * * *$ & - & 339,60 & - & \\
\hline Ratio especialistas formados & $0,54 * * *$ & 0,694 & 87,64 & 0,641 & $-0,452 * * *$ \\
\hline Ratio de camas & $0,21^{*}$ & 0,201 & 74,31 & 0,178 & $-0,034 * *$ \\
\hline Incremento población 1997-2007 & $-2,00 * * *$ & $-0,312$ & $-23,94$ & 0,165 & $-0,092 * * *$ \\
\hline Tasa de paro & $-5,46^{* * * *}$ & $-0,220$ & $-50,81$ & 0,134 & $-0,041 * * *$ \\
\hline Error estándar de la estimación & 32,78 & & & & \\
\hline $\mathrm{R}^{2} / \mathrm{R}^{2}$ ajustado & $0,846 / 0,833$ & & & & \\
\hline
\end{tabular}

$* \mathrm{p}<0,05 ; * * \mathrm{p}<0,01 ; * * * \mathrm{p}<0,001$ 
Tabla 6

Modelo de regresión lineal múltiple tras la exclusión de la variable de especialistas formados, y análisis de la influencia en el modelo de cada una de las variables incluidas

\begin{tabular}{|l|c|c|c|c|c|}
\hline \multirow{2}{*}{ Variables } & Modelo & \multicolumn{3}{c|}{ Valoración de la influencia de cada variable } \\
\cline { 2 - 6 } & Coeficientes & $\begin{array}{c}\text { (coeficiente } \\
\text { estandariz) }\end{array}$ & $\begin{array}{c}\text { Aportación media } \\
\text { al valor de la variable de } \\
\text { estudio }\end{array}$ & $\begin{array}{c}\mathbf{R}^{2} \text { del modelo } \\
\text { univariante }\end{array}$ & $\begin{array}{c}\text { Cambio en } \mathbf{R}^{2} \\
\text { al retirar la variable }\end{array}$ \\
\hline Constante & $321,93^{* * * *}$ & - & 321,93 & - & \\
\hline Tasa de estudios superiores & $10,94^{* * *}$ & 0,675 & 216,94 & 0,372 & $-0,439^{* * * *}$ \\
\hline Tasa pobl. mun. 10.000-500.000 H. & $-147,07^{* * *}$ & $-0,366$ & $-91,18$ & 0,131 & $-0,120^{* * * *}$ \\
\hline Incremento población 1997-2007 & $-1,77^{* *}$ & $-0,268$ & $-21,15$ & 0,084 & $-0,066^{* *}$ \\
\hline Error estándar de la estimación & 50,16 & & & & \\
\hline $\mathrm{R}^{2} / \mathrm{R}^{2}$ ajustado & $0,639 / 0,616$ & & & & \\
\hline
\end{tabular}

$* \mathrm{p}<0,05 ; * * \mathrm{p}<0,01 ; * * \mathrm{p}<0,001$.

$\mathrm{p}<0,001)$. Tampoco mostró una mejora significativa la incorporación al modelo de la variable cualitativa referida a la existencia o no de colegiación obligatoria (razón de verosimilitud 0,$9 ; \mathrm{p}>0,3)$.

\section{DISCUSIÓN}

Nuestro estudio confirma la existencia de una irregular distribución de los médicos en España, al comprobar que la ratio poblacional de profesionales en la provincia más dotada supera el doble del valor alcanzado por dicha ratio en la menos dotada, situación que, en líneas generales, se ha mantenido en niveles similares a lo largo de la pasada década. Dicha distribución, además, no es aleatoria, sino que se relaciona con distintas características sociodemográficas de las provincias, así como con su estructura sanitaria y su capacidad de formación de médicos y especialistas.

No se trata de un problema exclusivo de nuestro país ${ }^{22-24}$. En el ámbito europeo, $\mathrm{y}$ aunque los datos no son directamente comparables por referirse a médicos en activo, la razón que podemos obtener entre las regiones con mayor y menor ratio oscila entre 1,4 en los casos de Bulgaria, Polonia y Suecia, y valores superio- res a 3 en los casos de Rumania $(3,9: 1)$ y Holanda $(3,3: 1)^{25}$; hay que notar que el nivel regional de la estadística europea se corresponde con el español de CCAA. En el caso particular de Francia ${ }^{26}$, y a nivel departamental (más similar al nivel provincial usado en este estudio), la proporción entre las ratios extremas de médicos en activo es de 4,4:1 (2,3:1 si excluimos París, dada su desproporcionada dotación de médicos en relación al resto de departamentos), y su índice de Gini es de 0,16. En el caso de Alemania, donde se cuenta con información sobre total de médicos (tanto activos como inactivos) en los diferentes Länder (nivel regional, comparable al de nuestras CCAA), se obtienen una razón entre ratios extremas de 1,53 , y un índice de Gini de 0,08. Por todo ello, podemos concluir que la situación española, en lo que a distribución geográfica de los médicos se refiere, se encuentra dentro del rango de variabilidad existente en los países de nuestro entorno.

Tampoco su evolución en el tiempo muestra una variación lo suficientemente importante como para atribuir a problemas de distribución geográfica las dificultades recientemente surgidas a la hora de contratar profesionales en los distintos ámbitos geográficos. Es más, en el largo plazo dicha distribución parece haber 
mejorado, a tenor de los resultados que se pueden obtener de las cifras correspondientes al año $1972^{15}$ (coeficiente de variación 0,25 , razón entre las ratios máxima y mínima 2,58; ambos resultados, claramente superiores a los obtenidos con las cifras actuales).

Tanto el análisis de las correlaciones bivariadas como el de regresión lineal muestran una importante relación entre la ratio provincial de médicos y factores como la capacidad formativa o las características demográficas y socioeconómicas de las diferentes provincias, tal y como se había hipotetizado. Y es que, desde una perspectiva ecológica, la profesión médica no permanece ajena a la estructura de la sociedad, de la que forma parte $^{28}$. La varianza en la distribución de la ratio de médicos se reduce en casi dos tercios atendiendo exclusivamente a características sociodemográficas de las provincias, como la tasa de estudios superiores, la distribución de la población entre municipios de diversos tamaños y el incremento experimentado en la última década. La toma en consideración de la capacidad formativa de la provincia incrementa el ajuste de la predicción, tanto en el caso de la ratio de licenciados formados como, sobre todo, en el de la ratio de especialistas, con la que la varianza se reduce globalmente en más del $80 \%$.

En este sentido, los resultados de este trabajo van en la misma dirección que los hallazgos del realizado hace más de 30 años sobre una base analítica similar ${ }^{15}$, obteniendo un mayor poder predictivo, probablemente al analizar la capacidad formativa de cada provincia desde una perspectiva más amplia, que incluye la formación de médicos residentes, y al hacerlo de forma acumulativa, al considerar el número total de médicos y de especialistas formados en la misma, mientras que el estudio referido lo hace de forma puntual en un momento dado. Ello probablemente influye en que el gradiente norte-sur de la distribución de médicos quede prácticamente explicado mediante las variables utilizadas, dado el efecto absolutamente marginal (y estadísticamente no significativo) que añade la variable longitud geográfica.

Parece, además, confirmarse un cierto fenómeno "inercial" en la distribución de los servicios, tanto por la correlación inversa entre crecimiento poblacional y las ratios de unidades escolares y de médicos, como por la inclusión de esa variable en los modelos de regresión, también con signo negativo. Este aspecto es de gran importancia, ya que la previsión de la dinámica poblacional podría facilitar, de confirmarse ese efecto inercial, la planificación de los recursos humanos profesionales, adaptando su evolución a la de la propia población a la que deben servir.

La utilización de los datos de colegiación no está exenta de dificultades, debido a la recogida descentralizada de los mismos por colegios provinciales y la no obligatoriedad de la colegiación en Andalucía, Canarias, Asturias y Extremadura, aunque no hemos encontrado una influencia significativa del hecho de la obligatoriedad o voluntariedad de la colegiación en la variable principal, ni en el análisis bivariado ni en el multivariado. Pero al no existir un registro vivo y fiable de profesionales que refleje la imagen real de la profesión tanto a nivel cuantitativo como cualitativo (situación de actividad, distribución por especialidades, etc) $)^{6,29}$, son los únicos datos oficiales sobre el número y la distribución de los médicos en España, ofrecidos con periodicidad anual. Las posibles fuentes alternativas ofrecen menos garantías para este fin, bien por no recoger a todos los profesionales ${ }^{10}$, bien por no poder ubicarles correctamente ${ }^{20}$. Estas mismas circunstancias hacen que el análisis de la distribución por especialidades, o por grandes grupos de las mismas, entrañe gran dificultad y requiera un 
aparato crítico específico, digno de desarrollarse en otros trabajos, dados los diferentes patrones de distribución de las especialidades (en el que los especialistas parecen concentrarse en torno a los grandes dispositivos asistenciales) $)^{30,31}$ y su posible influencia en la calidad de la asistencia sanitaria ${ }^{32}$.

Hemos optado por considerar la cifra total de profesionales, pues el dato sobre profesionales jubilados no tiene por qué corresponderse con el de inactivos, y la localización de aquéllos también puede expresar las dinámicas geográficas de la profesión, sin más limitación que la correspondiente a una mayor antigüedad de las mismas. Atender a la distribución por edad escapa de los objetivos del presente estudio; pero dada la importancia que dicha distribución puede tener en la capacidad de recambio de los profesionales, podría ser un buen objetivo para sucesivos trabajos en este ámbito, en el que, por otro lado, ya se han hecho algunas incursiones, aunque sólo a nivel descriptivo y de Comunidad Autónoma ${ }^{10,20}$.

El diseño del estudio no permite el establecimiento de inferencias causales. No parece que la ratio de médicos pueda influir de forma importante en los indicadores socioeconómicos y demográficos de una determinada provincia, por lo que es fácil aceptar que la influencia es inversa. En el caso de la capacidad formativa de médicos no se puede asumir fácilmente una influencia causal sobre la ratio de médicos, dado que también puede esperarse cierta causalidad en sentido inverso. Sin embargo, la relación mostrada en la literatura internacional entre lugar de ejercicio y lugar de formación ${ }^{30-34}$ sí parece apuntar a que dicha influencia existe, y ha de tenerse en consideración.

Si la capacidad formativa de cada provincia puede actuar como fenómeno compensatorio tanto de la desigualdad derivada de las circunstancias socioeconómicas como de la evolución de las cifras poblacionales, la planificación de recursos humanos dispondría de una importante herramienta para influir en la distribución de los profesionales, a considerar junto a otras medidas tendentes a optimizar el número total de los mismos y la distribución de competencias y responsabilida$\operatorname{des}^{3,4}$. Se hace necesario, por tanto, profundizar mediante nuevos estudios en esta posible influencia causal, para posteriormente poder emprender acciones concretas destinadas a reducir la desigualdad en dicha distribución, como ya se está haciendo en otros países ${ }^{35}$. Mientras tanto, concentrar la formación en ámbitos geográficos con una ratio de profesionales ya de por sí elevada no parece lo más adecuado.

\section{BIBLIOGRAFÍA}

1. Joint Learning Initiative. Human resources for health. Overcoming the crisis [Monografía en Internet]. Cambridge: Harvard University Press; 2004. [Citado 12 de noviembre de 2005]. Disponible en http://www.globalhealthtrust.org/Report.html.

2. Simoens S, Hurst J. Matching supply with demand for the services of physicians and nurses. En: The OECD Health Project. Towards high performing health systems. Policy studies. Paris: OECD Publications; 2004.

3. Jiménez Palacios A. La coordinación de políticas de recursos humanos (editorial). Rev Adm Sanit. 2008; 6(3): 367-70.

4. Gérvas J, Bonis J, Seminarios de Innovación en Atención Primaria 2007. El debate profesional acerca de la escasez de médicos. Rev Esp Salud Pública. 2008; 82(6): 627-35.

5. Rodríguez JA. Estructura de la profesión médica española. Reis: Revista Española de Investigaciones Sociológicas 1987; 32: 141-66.

6. Maynard A, Amaya C, Coronado A, Grau T, Alcaide PL. El número de médicos en España. Análisis de una situación crítica. $2^{\mathrm{a}}$ ed. Madrid: Fundación CESM; 1999.

7. González B. El mercado laboral sanitario y sus consecuencias en la formación. Numerus clausus. En: Fundación BBV. La formación de los profe- 
sionales de la salud. Escenarios y factores determinantes. 2a ed. Bilbao: Fundación BBV; 1998. pp. $429-467$.

8. Pedraza V. Recursos humanos del sistema de salud: una reforma pendiente. Med Clin (Barc). 2001; 116: 779-81.

9. García MA, Amaya C. ¿Faltan médicos en España? Ars Medica. Revista de Humanidades. 2007; 6(2): 152-70.

10. González B, Barber P. Oferta y necesidad de médicos especialistas en España (2006-2030) [Monografía en Internet]. Madrid: Ministerio de Sanidad y Consumo; 2007. [Último acceso, 12 de julio de 2008] Disponible en http://www.msc.es/novedades/docs/necesidadesEspeciales06_30.pdf

11. Rodríguez Sendín JJ. Necesidades de médicos en España: a propósito de estudios, debates y posibles soluciones [Monografía en Internet]. Madrid: Organización Médica Colegial de España; 2007. Disponible en http://www.cgcom.org/sites/default/ files/07_11_29_sendin_demografia.pdf

12. González B, Urbanos RM, Ortega P. Oferta pública y privada de servicios sanitarios por comunidades autónomas. Gac Sanit. 2004; 18 (supl 1): 82-9.

13. Ortiz T. Médicos en la Andalucía del siglo XX. Número, distribución, especialismo y participación profesional de la mujer. Granada: Fundación Averroes; 1987.

14. Saturno Hernández PJ. Los médicos de la comarca de la Huerta de Murcia. Número, distribución espacial, movilidad, grado de especialización de 1960 a 1974. Asclepio 1981; 33: 23-80.

15. González B. El capital humano en el sector sanitario: la distribución de médicos en España. Madrid: Centro de Investigaciones Sociológicas; 1979.

16. Instituto Nacional de Estadística [Base de datos en Internet]. Madrid: Instituto Nacional de Estadística; 2008. [Último acceso, 30 de octubre de 2008]. Disponible en www.ine.es

17. Ministerio de Sanidad y Consumo. Catálogo de Centros de Atención Primaria del SNS y Catálogo Nacional de Hospitales [base de datos en Internet]. Madrid: Ministerio de Sanidad y Consumo; 2008. [Último acceso, 28 de julio de 2008]. Disponible en http://www.msc.es/ciudadanos/prestaciones/centrosServiciosSNS/hospitales/home.htm

18. Ministerio de Educación y Cultura, Subdirección General de Títulos, Convalidaciones y Homologa- ciones. Estadísticas de Títulos Universitarios. Ministerio de Educación y Cultura; 1999.

19. Ministerio de Ciencia e Innovación, Secretaría General del Consejo de Coordinación Universitaria. Estadística del alumnado [base de datos en Internet]. [Último acceso, 17 de septiembre de 2008]. Disponible en http://www.micinn.es/univ/ jsp/plantilla.jsp?area $=$ ccuniv\&id=E125

20. Amaya C, García MA. Demografía médica en España. Mirando al futuro. Madrid: Fundación CESM; 2005.

21. Castillo-Salgado C, Schneider C, Loyola E, Mújica O, Roca A, Yerg T. Medición de desigualdades en salud: coeficiente de Gini e índice de concentración. Boletín Epidemiológico / OPS [Internet] 2001 [último acceso, 3 de septiembre de 2008]; 22(1): 3-4. Disponible en http://www.paho.org/ spanish/sha/BE_v22n1.pdf

22. Zurn P, Dal Poz MR, Stilwell B, Adams O. Imbalance in the health workforce. Human Resources for Health [Internet] 2004 [último acceso, 13 de septiembre de 2008]; 2:13. Disponible en http://www.humanresources-health.com/content/2/1/13

23. Council on Graduate Medical Education (COGME). Tenth Report: Physician Distribution and Health Care Challenges in Rural and InnerCity Areas [Monografía en Internet]. U.S. Department of Health and Human Services; 1998. [Último acceso, 15 de septiembre de 2008]. Disponible en http://www.cogme.gov/10.pdf

24. Pong RW, Pitblado JR. Geographic Distribution of Physicians in Canada: Beyond How Many and Where [Monografía en Internet]. Ottawa: Canadian Institute for Health Information; 2005. [Último acceso, 8 de septiembre de 2008]. Disponible en http://www.theruralcentre.com/CIHI\%20\%20Geographic\%20Distribution\%20of\%20Physicians $\% 20$ in $\% 20$ Canada.pdf

25. EUROSTAT, the Statistical Office for the European Communities. Regional and urban statistics [Base de datos en Internet]. EUROSTAT ; 2008. [Ultimo acceso, 11 de septiembre de 2008] Disponible en http://epp.eurostat.ec.europa.eu/portal/page?_pagei $\mathrm{d}=1335,72265683,1335 \_72320396 \&$ dad=portal\&_schema=PORTAL

26. Ordre National des Médecins. L'atlas de la démographie medicale en France. Situation au 1er janvier 2007 [Monografía en Internet]. París: Conseil National de 1'Ordre ; 2007. [Último acceso, 12 de septiembre de 2008]. Disponible en http://www.web.ordre.medecin.fr/demographie/a nnexesatlas2007.pdf 
27. Bundesärztekammer (Cámara de Médicos de Alemania). Ärztestatistik der Bundesärztekammer zum 31.12.2007 [Monografía en Internet]. Bundesärztekammer; 2008. [Último acceso, 15 de septiembre de 2008]. Disponible en Internet, http://www.bundesaerztekammer.de/page.asp?his $=0.3 .6097$

28. Anderson JG, Marshall HH. The structural approach to physician distribution: a critical evaluation. Health Serv Res. 1974; 9(3): 195-207.

29. González B, Barber P. Dificultades, trampas y tópicos en la planificación del personal médico. Gac Sanit. 2008; 22:393-5.

30. Niel X. Les facteurs qui influencent la demographie medicale au niveau regional. Cah Socio Démo Méd. 2001; 41(2): 141-72.

31. Couffinhal A, Mousqués J. La démographie médicale française: état des lieux. Questions d'Economie de la Santé [Internet] 2001;44:1-6. Disponible en
http://www.irdes.fr/Publications/Bulletins/QuestEco/pdf/qesnum44.pdf

32. Starfield B, Shi L, Grover A, Macinko J. The effects of specialist supply on populations' health: assessing the evidence. Health Aff (Millwood). [Internet] 2005; W5:115-8 [web exclusive]. Disponible en http://content.healthaffairs.org/cgi/reprint/hlthaff.w5.97v1

33. Kristiansen IS, Forde OH. Medical specialists' choice of location: the role of geographical attachment in Norway. Soc Sci Med. 1992; 34(1): 57-62.

34. Mathews M, Rourke JTB, Park A. National and provincial retention of medical graduates of Memorial University of Newfoundland. CMAJ. 2006;175(4):357-60.

35. Berg AO, Norris TE. A workforce analysis informing medical school expansion, admissions, support for primary care, curriculum and research. Ann Fam Med. 2006 4: S40-44. 
